\title{
Label-free optical imaging technologies for rapid translation and use during intraoperative surgical and tumor margin assessment
}

Stephen A. Boppart

J. Quincy Brown

Camile S. Farah

Esther Kho

Laura Marcu

Christobel M. Saunders

Henricus J. C. M. Sterenborg 


\title{
Label-free optical imaging technologies for rapid translation and use during intraoperative surgical and tumor margin assessment
}

\author{
Stephen A. Boppart, ${ }^{a, \star}$ J. Quincy Brown, ${ }^{b}$ Camile S. Farah, ${ }^{c}$ Esther Kho, ${ }^{d}$ Laura Marcu, ${ }^{e}$ \\ Christobel M. Saunders, ${ }^{f}$ and Henricus J. C. M. Sterenborg ${ }^{d, g}$ \\ a University of Illinois at Urbana-Champaign, Beckman Institute for Advanced Science and Technology, Urbana, Illinois, United States \\ ${ }^{b} T u l a n e$ University, Department of Biomedical Engineering, New Orleans, Louisiana, United States \\ 'University of Western Australia, UWA Dental School, Oral Health Centre of Western Australia, Discipline of Oral Oncology, Nedlands, \\ Western Australia, Australia \\ dNetherlands Cancer Institute, Department of Surgery, Amsterdam, The Netherlands \\ eUniversity of California-Davis, Department of Biomedical Engineering, Comprehensive Cancer Center, Davis, California, United States \\ 'The University of Western Australia, Department of Surgical Oncology, Crawley, Western Australia, Australia \\ ${ }^{9}$ Academic Medical Center, Department of Biomedical Engineering and Physics, Amsterdam, The Netherlands
}

\begin{abstract}
The biannual International Conference on Biophotonics was recently held on April 30 to May 1, 2017, in Fremantle, Western Australia. This continuing conference series brought together key opinion leaders in biophotonics to present their latest results and, importantly, to participate in discussions on the future of the field and what opportunities exist when we collectively work together for using biophotonics for biological discovery and medical applications. One session in this conference, entitled "Tumor Margin Identification: Critiquing Technologies," challenged invited speakers and attendees to review and critique representative label-free optical imaging technologies and their application for intraoperative assessment and guidance in surgical oncology. We are pleased to share a summary in this outlook paper, with the intent to motivate more research inquiry and investigations, to challenge these and other optical imaging modalities to evaluate and improve performance, to spur translation and adoption, and ultimately, to improve the care and outcomes of patients. ๑ 2017 Society of PhotoOptical Instrumentation Engineers (SPIE) [DOI: 10.1117/1.JBO.23.2.021104]
\end{abstract}

Keywords: optical imaging; label-free; cancer surgery; surgical margins.

Paper 170508SSR received Aug. 1, 2017; accepted for publication Nov. 28, 2017; published online Dec. $29,2017$.

\section{Microscopic Assessment of the Tumor Margin and Microenvironment is an Important Surgical and Medical Challenge that Can be Addressed with Optical Imaging Technologies}

Label-free optical imaging offers the potential to microscopically assess margins in surgical oncology, and various technologies were presented and critiqued at the 2017 International Conference on Biophotonics. ${ }^{1}$

The surgical resection of solid tumors can be an effective, even curative, treatment and is often combined with adjuvant chemotherapy or postoperative radiation therapy. ${ }^{2}$ In the process of removing a solid tumor, there are several margins of interest, and the evaluation of these margins is critical for treatment management decisions, prognosis, and positive outcomes. A "surgical margin" is created when the surgeon cuts tissue and removes the tumor mass. The surface of this mass ideally includes a region of normal tissue in order to ensure no tumor is left behind in the surgical cavity. Following resection of this mass, a resection cavity or resection bed now exists in the patient, and this cavity surface is the in vivo counterpart to the surgical margin. The "tumor margin" represents the boundary between the tumor

*Address all correspondence to: Stephen A. Boppart, E-mail: boppart@illinois .edu and the surrounding normal tissue. Ideally, this tumor margin should lie fully within the resected mass and be deeper than the surgical margin or surface of the mass. Therefore, the primary challenge for ensuring negative or tumor-free surgical margins following resection is that the microscopic boundary of malignancy is, typically, neither visually apparent nor grossly identifiable via palpation or other means by the surgeon.

Intraoperative pathology techniques that rely upon a surgeon-directed collection of small tissue samples from the edge of the resected cavity for analysis have, in general, provided disappointing sensitivity for detection of tumor at the surgical margin (a positive margin), particularly for organs with large resection specimens. That this would be the case is logical, given the difficulties in visual discrimination of the microscopic tumor boundary or small foci of tumor cells. Furthermore, even permanent "bread-loafed" pathology, conducted within days of the surgery, still represents an undersampling technique, in which the majority of the specimen surface (or surgical margin) goes unexamined microscopically, which could have significant implications in adjuvant decision-making. Given the difficulties in heuristic selection of appropriate sampling sites for detection of residual tumor at the surgical margin, one approach is to cast the widest net possible in terms of examination of the ex vivo surgical margin or the in vivo resection bed by advancing technologies capable of imaging the entire surface of en bloc resections, with the resolution necessary to resolve nuclear atypia or other architectural or molecular features of tumor. Imaging of 
resected specimens in this context includes not only large widefield imaging but also imaging based on rapidly scanning an optical beam focused to either a point or a line. While labelfree imaging techniques rely inherently on the spatial resolution of the optical system, the concomitant use of molecular probes allows for subresolution detection of molecular or cellular targets. Such approaches reduce or eliminate the risk that microscopic or occult tumor at the surgical margin will remain undetected (either intraoperatively or postoperatively), although they come at the price of (a) the need for very high-throughput imaging technologies and (b) the need for an accordingly highthroughput means of analyzing the resulting copious image data.

While it may be ideal to microscopically image and analyze the entire surgical margin of a resected specimen and/or image the entire in vivo resection bed (Fig. 1), no imaging technology currently exists to effectively ensure complete resection of tumor at the cellular level. Frozen section histopathology, a procedure in which small portions of resected tissue specimens are rapidly frozen, sectioned, and stained for microscopic evaluation during a surgical procedure, often suffers from poor tissue section quality and significantly undersamples the full surgical margin due to time constraints. ${ }^{3} \mathrm{X}$-ray specimen radiography uses a portable planar x-ray system often located immediately outside the operating room to image resected tumor/tissue specimens to ensure that the radiologically denser tumor and tumor margin lie within the surgical margin or surface of the specimen. However, the relatively low resolution and lack of three-dimensional (3-D) imaging capabilities make it difficult to find microscopic disease

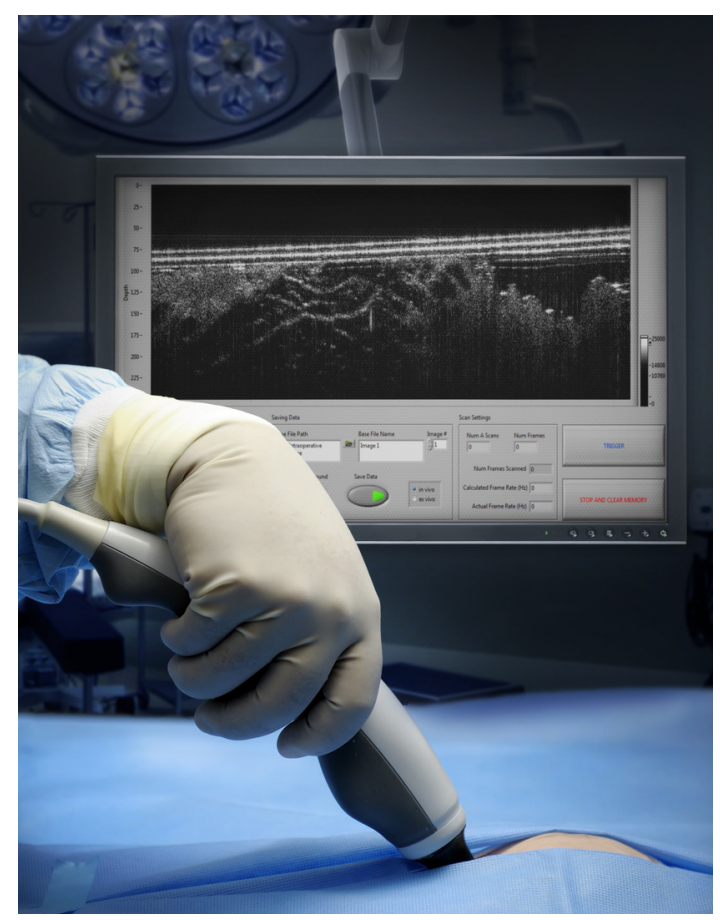

Fig. 1 Simulated representative intraoperative imaging scenario using an optical imaging-based hand-held probe for assessing the tumor cavity and resection bed. Advances are needed toward slide-free, stain-free, real-time, in vivo imaging during surgery to reduce positive margin and reoperation rates and to improve patient outcomes. Figure courtesy of Stephen Boppart, the Biophotonics Imaging Laboratory, and the Imaging Technology Group at the Beckman Institute for Advanced Science and Technology, University of Illinois at Urbana-Champaign, Urbana, Illinois. at the surgical margins. ${ }^{4}$ Touch prep cytology relies on the sloughing of tumor cells from the surgical margin, which are then collected on glass microscope slides for staining and microscopic evaluation. ${ }^{5}$ However, comprehensive sampling of the surgical margin is difficult, and adherent or subsurface tumor cells are not identified. Outside of the optical spectrum, an intraoperative surgical probe that relies on changes in the radiofrequency spectrum between normal and tumor-bearing tissue is being used for margin assessment, ${ }^{6}$ but given the relatively large area that is measured at each point location of the probe, small foci of tumor cells within those measured volumes cannot readily be detected. This device also emits an indicator for the presence of a positive surgical margin, rather than generating an image for interpretation.

A major class of intraoperative optical imaging technologies for margin assessment relies on the use of contrast agents or molecular probes that are either applied topically to the surface of the resected specimen or the in vivo resection bed, or injected intravenously to circulate and localize to the tumor. ${ }^{7,8}$ Aside from the required drug approvals for these probes, their use is challenged by the need for high site-specific targeting, low nonspecific binding to normal cells and tissues, often a darkened surgical suite to collect weak optical signals, and the pharmacokinetics for the administration, targeting, and clearance. ${ }^{9,10}$ While these probe- or label-based methods continue to show promise, we believe there is significant merit in pursuing labelfree optical imaging methods to address this critical surgical need.

Historically, the majority of new imaging technologies for this application have been developed for examining and assessing the resected tissue specimen for more rapid intraoperative analysis, followed by the current gold-standard postoperative examination of hematoxylin and eosin (H\&E)-stained or immunohistochemically stained tissue sections by a pathologist, which occurs days after the surgical procedure. To date, technologies have focused on ascertaining if any tumor cells are near the edge of the excised specimen, indicating that there may be some residual disease left behind in the patient. Perhaps a more important surgical goal is to directly ensure that no tumor cells are left behind by examining the surgical cavity for residual tumor, not the excised specimen. Therefore, there is a clear need for advancing optical imaging technologies that have the potential for real-time microscopic imaging of the in vivo resection bed. To more rapidly translate optical imaging technologies for surgical use, it becomes imperative that technologies enable label-free contrast at microscopic cellular resolution, and can be used in vivo and in real time. These technologies must also offer sufficiently high throughput so that large surface and subsurface areas of tissue can not only be imaged rapidly during the surgical procedure, but also be assessed both visually and with computer-aided-diagnosis (CAD) algorithms that screen, analyze, and flag tissue sites that are positive or suspicious for residual disease.

The emerging field of "digital pathology" is based on hardware technology and software algorithms for optically scanning a standard histology slide to capture a large, high-resolution, multiscale, digital image of a stained tissue section on a glass microscope slide. ${ }^{11}$ Recently, the U.S. FDA approved the first digital pathology system for doing this, enabling pathologists to read, interpret, and make clinical diagnoses strictly from these digital images (while keeping the glass microscope slide available for any problematic findings seen on the digital 
image). ${ }^{12}$ While this is certainly an advance for pathology, perhaps an even more compelling need exists for more advanced imaging technologies to catapult the field of pathology toward slide-free stain-free digital histology captured in vivo and in real time. Such optical imaging technologies, which were the focus of this conference session, ${ }^{1}$ not only offer the potential to improve upon surgical oncology practices and surgical tumor margin assessment, but also offer potentially new directions in the field of pathology by reducing or potentially obviating the need for the labor-intensive, time-consuming, and costly process of standard histopathology to generate stained slides. Here, we highlight an incomplete yet representative set of optical imaging technologies that offer the potential for slide-free, stain-free, real-time, in vivo imaging of the tumor resection bed, as well as the surgical margin of the resected specimen. We will use breast cancer and breast conserving surgery as one example where these technologies offer the potential for impact and improvements in patient care.

\section{Breast Cancer Surgery: a Surgical Scenario in Need of Intraoperative Imaging Solutions}

Over 1.8 million women worldwide are diagnosed with breast cancer each year. In many developed countries, $70 \%$ will now undergo breast conserving surgery and $30 \%$ of tumors will be screen-detected, often very small and impalpable. While this represents good news for women with breast cancer, this represents a challenge for surgeons, as small tumors cannot be felt and thus must be localized by a variety of methods to guide excision. Roughly, one-third of patients will require a second surgery to ensure that the entire tumor has been removed. Thus, the challenges to overcome for breast surgeons include: (1) to know the exact extent of the lesion before surgery, (2) to have a sure-fire and quick technique to localize the lesion for surgery, (3) to be able to detect exactly how much tissue to remove at surgery and how much to leave behind, and (4) to do this in a single operation to achieve the best cosmetic as well as oncological outcome for the patient.

The optical imaging techniques described in this paper have the potential to address the third and fourth challenges identified, whereas advances in other biomedical imaging and sensing technologies have the potential to impact the first and second challenges. All of this may also need to be achieved in the more challenging situation after neoadjuvant therapy and before postoperative radiation therapy, and with what is morphologically very heterogeneous tumor and host tissue. If this is not achieved, outcomes can lead to inadequate margins with up to a $30 \%$ reoperation rate, ${ }^{13}$ higher costs, longer operation times, longer wait lists, worse cosmesis, higher mastectomy rates, higher doses of radiation therapy, missed lesions, increased local recurrence, and potentially a poorer survival rate.

Current techniques for margin assessment in breast cancer have included intraoperative pathology assessment, but to date, this has not been shown to change re-excision rates. Decker et al. ${ }^{14}$ completed a study of 8259 cases of ductal carcinoma in situ with intraoperative pathology assessment using frozen section or touch prep cytology that still resulted in a high $26 \%$ re-excision rate. From a surgeon's perspective, we need not so much to assess tumor margins as to assess residual disease in the surgical cavity at the micrometer to millimeter scale. This needs to be easily interpretable by the surgeon in real time, be ergonomic, quick, and at high resolution. Most of all, the method and technology need to be accurate, thus validated against the gold standard of histology, and of course, improve patient outcomes. The following sections review some of the current optical imaging technologies that are being developed to address these needs.

\section{High-Resolution Wide Field-of-View Surface Imaging of Tumor Resection Margins: Addressing the Need for Large-Area Coverage}

Several examples of technologies that have pushed the boundaries for imaging of large areas of fresh resected tumor specimens with high throughput and high resolution exist in the recent literature. Following on prior developments of rapid stripmosaicking fluorescence confocal microscopy, ${ }^{15-17}$ Abeytunge et al. ${ }^{18}$ demonstrated imaging of $4-\mathrm{cm}^{2}$ fresh breast tissue specimens within $10 \mathrm{~min}$ at subcellular resolution. Accurate identification of varying types of breast malignancies was demonstrated, and the study was enhanced by clinically relevant data analysis in which entire images were reviewed (rather than a selection of smaller ROIs) and compared to standard histology. Iftimia et al. ${ }^{19}$ demonstrated the possibility of combining OCT as a fast, low-resolution technique for screening for suspicious areas in large specimens with confocal microscopy, a slower but higher resolution technique for detailed analysis of suspicious areas. This is an attractive strategy that can seamlessly integrate the technical advantages of multiple techniques while minimizing the practical limitations of each. Giacomelli et al. ${ }^{20}$ and Yoshitake et al. ${ }^{21}$ demonstrated the use of confocal and multiphoton microscopy for virtual H\&E imaging of excised sliced breast specimens, enabling determination of various breast pathologies. Glaser et al. ${ }^{22}$ recently demonstrated volumetric imaging of fresh slices of breast and prostate tissue immersed in silicone oil using light sheet microscopy. This method achieved rapid large-area imaging at a modest depth of $50 \mu \mathrm{m}$ in fresh tissue specimens; however, due to the image detection plane being oriented at $45 \mathrm{deg}$ relative to the specimen surface, the number of pixels per image frame that corresponded to the actual specimen surface was not maximized, compared to en-face approaches. Using en-face structured illumination microscopy, Wang et al. ${ }^{23}$ imaged the circumferential surface (true surgical margin) of freshly excised radical prostatectomy specimens without any slicing, compression, liquid immersion, or other physical manipulation of the specimens. An imaging throughput rate of 18 megapixels per second of surface image data at $1.3-\mu \mathrm{m}$ resolution was achieved, with the imaging speed limited by the microscope stage movement and not by the raw imaging throughput, which was capable of $>130$ megapixels per second. In an initial 24-patient series of radical prostatectomy specimens imaged immediately after excision, a per-patient average (range) of 11.7 (3.1 to 16.1) gigapixels of image data covering 47.3 (12.6 to 64.8$) \mathrm{cm}^{2}$ of tissue surface area in surgically relevant timeframes was achieved, and the ability to identify otherwise occult areas of residual tumor on the tumor margin by blinded pathology review of the full surface images was demonstrated. The technique can be combined with dual-stain strategies to enable virtual H\&E rendering of the tissue surface. ${ }^{24}$

Each of the above techniques represent promising approaches for large-area, high-resolution, fresh tissue imaging that could find routine clinical application for the detection of residual tumor at the resection margin with potentially higher 
sensitivity compared to surgeon-directed sampling approaches. Imaging of the excised specimen, analogous to the gold standard in terms of image contrast and resolution, albeit with greatly improved performance, will likely encounter low-to-modest regulatory barriers that could see these technologies clinically translated in the relative short term. Imaging studies that examine the uncut, en-face surface of excised specimens, rather than bread-loafed slices, most closely represent the intended clinical use scenario and should be prioritized. However, this introduces challenges in terms of validation against gold-standard histology, since physical-sectioning histology used for accuracy validation must be performed on cross sections of the tissue due to technical limitations. This is a challenge for which enabling developments are welcomed. Additionally, each technique also generates image data on the scale of hundreds of megapixels to tens of gigapixels per patient. If the amount of image data that must be reviewed by on-call pathologists is increased beyond practical limits, efforts to increase image collection speed could be undermined by image interpretation bottlenecks. Thus, strategies for computer-assisted searches of the image data (similar to those currently leveraged in automated Pap smear screening) should be pursued in parallel with instrumentation advances to ease eventual clinical translation.

\section{Hyperspectral Imaging for Intraoperative Margin Assessment: Addressing the Need for Label-Free Contrast during Surgery}

Hyperspectral imaging as a technique for assessment of resection margins during surgery can be performed both in vivo and ex vivo. In vivo imaging may be the ultimate goal but requires a complicated instrument due to usage in a sterile environment and the complicated imaging geometry of a surgical cavity. A less-demanding approach is ex vivo imaging of the surgical specimen, where imaging can be performed directly after excision but still in the operating room in a controlled imaging geometry. Since feedback from hyperspectral imaging can be produced within minutes after resection, it can still be used to give real-time feedback during the procedure and help the surgeon to re-excise suspected regions and reduce reoperations.

In previous work using fiber-optic diffuse reflection spectroscopy in the near-infrared (NIR) region, excellent results were obtained for distinguishing cancer from normal tissue with high accuracies. ${ }^{25-27}$ With the chosen distances between source and detector fibers, the sampling volume of this method is mainly determined by the fiber-optic geometry and is on the order of a few $\mathrm{mm}^{3}$ or less. As this is on the order of the transport mean free path, inhomogeneities in the tissue will average out. The measured spectra can thus be analyzed accurately using diffusion theory and translated to concentrations of chromophores, such as water and fat. ${ }^{28}$ For evaluating a larger surface, such a point measurement procedure would be unpractical and too time-consuming. Therefore, an approach based on hyperspectral imaging has been developed, focusing on the wavelength range from 950 to $1640 \mathrm{~nm}$. In a diffuse imaging geometry, the sampling volume is determined by the optical properties of the tissue. This sampling volume can reach up to $1 \mathrm{~cm}^{3}$ and varies strongly with wavelength. Due to these large sampling volumes that also vary, and the high inhomogeneity of breast tissue, the previously described spectral analysis, based on diffusion theory, is no longer valid. Thus, other approaches to analyze the data have to be developed, such as spectral derivative imaging, ${ }^{29}$ and more statistical approaches, such as support vector machines, linear and logistic regression, neural networks, and decision trees.

In studies involving ex vivo imaging, instead of measuring the surgical margin of the excised specimen, 5-mm-thick slices were cut by the pathologist during standard histopathological evaluation. The tissue slices that contained both tumor and healthy tissue were selected for hyperspectral measurements. After the hyperspectral measurements were made, H\&E-stained histology slides of the slices were obtained for correlations. This, in principle, enables a one-to-one comparison between the hyperspectral image and the pathology slide of the same tissue. This approach allowed measurements in a controlled setting, generating a large hyperspectral database. With this database, it then becomes possible to develop an algorithm that could be used to analyze the hyperspectral images of the surgical margins.

The main problem encountered in this approach was the difference in shape between the pathology slide and the hyperspectral image. This is a result of the manipulation of these mechanically very flexible specimens during the standard histopathological protocol. This problem was partially solved by overlaying a regular color image, obtained along with the hyperspectral image, over the hyperspectral image, and digitally modifying the shape of the pathology slide to this color image on the basis of visual landmarks. This is a manual procedure, and the match with the pathology slide had some errors, limiting the accuracy. To prevent these matching errors and increase the quality of the training data for the classification algorithm, distinctions between "border pixels," obtained at tissue type borders, and "sure pixels," pixels surrounded by pixels with the same tissue type, were made. As pixel size was typically $<1 \mathrm{~mm}$, by excluding the border pixels, it was possible to also exclude the smaller pockets of cancer that were $<2 \mathrm{~mm}$ in size. Algorithms were, therefore, trained using only sure pixels. The evaluating algorithms on a dataset with sure pixels yielded excellent results with accuracies over 97\%. However, when including the border pixels in the evaluation, the results dropped dramatically, especially for DCIS. These smaller pockets of tumor tissue are the most difficult to detect and are the main cause of positive surgical margins. Positive identification of these small pockets of cancer may be the most important requirement for an intraoperative technique to impact the success of the procedure. Hyperspectral imaging in the NIR region has the potential to be used to accurately identify positive surgical margins within minutes after resection. The remaining challenge, however, is to develop an experimental approach to validate the accuracy for small pockets of cancer.

\section{Fluorescence Lifetime Imaging Microscopy for Surgical Imaging, Guidance, and Augmented Reality: Addressing the Need for Metabolic Imaging, More Than Just Structural Imaging}

Carcinogenic biochemical alterations of tissues and cells (proximal to a tumor mass) typically occur prior to morphological changes. Moreover, morphological features may not reflect the tumor metabolism - an important aspect in clinical management of patients with cancer. While a milestone in the field of diagnostic imaging has been the integration of structural and functional modalities, such as combined PET-CT and PETMRI, these multimodality systems can only be employed prior to surgical interventions. ${ }^{30}$ Optical techniques that make 
use of nonionizing radiation offer the potential to assess functional and molecular features in pathophysiological conditions during interventions. Tissue autofluorescence properties, in particular, offer a means to evaluate biochemical and metabolic changes in biological tissues. A range of endogenous tissue fluorophores provide contrast mechanisms for detecting and quantitatively evaluating these changes. This includes, but is not limited to, the matrix protein collagen involved in tumor microenvironment remodeling, and nicotinamide adenine dinucleotide (NADH) and flavin adenine dinucleotide (FAD) associated with cellular metabolism. ${ }^{31}$

Increasingly, there is an interest in exploiting fluorescence lifetime contrast to analyze tissue autofluorescence properties, and in particular, as a means for intraoperative diagnostics and surgical guidance. While autofluorescence can be measured or imaged using conventional intensity measurements, it is challenging to make sufficiently quantitative measurements for diagnostic applications in the surgical field, since the autofluorescence intensity signal can be affected by fluorophore concentration, variation in the temporal and spatial properties of the excitation light, the detection efficiency, and attenuation by light absorption and scattering within the tissue. In contrast, fluorescence lifetime measurements are inherently ratiometric and, therefore, minimally affected by the many factors that can compromise autofluorescence intensity measurements. ${ }^{32}$ The use of lifetime-based techniques in clinical settings has been hampered, however, by the need of complex instrumentation and computational methods to measure and analyze the autofluorescence signals, respectively. Recent advances in laser light sources, fast detectors, and electronics have enabled an improved technological implementation of fluorescence lifetime imaging systems in terms of higher performance, lower cost, and more compact and ergonomic instrumentation compatible with clinical environments. A variety of instrumentations used for fluorescence lifetime measurements have evolved and are being used experimentally in clinical settings, including single-channel (point) spectroscopy utilizing ultrafast sampling, time-correlated single-photon counting, and fluorescence lifetime imaging microscopy (FLIM) based on time-gated imaging camera. $^{32}$

For example, one fluorescence lifetime imaging (FLIM) technique was presented at the 2017 International Conference on Biophotonics. This technique is currently being extensively evaluated in patients undergoing surgery for tumor removal as a potentially new means for intraoperative real-time diagnostics, surgical guidance, and augmented reality. ${ }^{33,34}$ The FLIM device is based on a point-spectroscopy multispectral time-resolved pulse-sampling technique, and FLIM images are constructed by scanning the fiber-optic probe above the area of interest. The instrument incorporates unique features, including the ability to (1) rapidly time-resolve the fluorescence emission in multiple spectral wavelength bands (e.g., four channels) and display the derived optical parameters in $<1 \mathrm{~ms}$ per data point, (2) augment in real time the computed fluorescence decay parameters on the white-light (WL) images of the surgical field of view, (3) account for sudden variation in fluorescence intensity though a dynamic detector gain control scheme, and (4) track the tissue maximum permissible exposure to laser excitation flux. Current applications of this FLIM technique include delineation of surgical margins in head and neck cancer, in particular, during trans-oral robotic surgery, brain tumor margin delineation during craniotomy procedures and guided biopsy, and intraoperative detection of positive margins during breast conserving surgery procedures. Current results demonstrate that FLIM-derived parameters may allow not only for an accurate delineation of tumor from surrounding normal tissue but also for resolving biochemical heterogeneity within a particular tissue type. Moreover, the current FLIM system has the potential to be easily integrated in the operating room workflow as well as with surgical robots-such as the da Vinci ${ }^{\circledR}$ surgical system.

While the main technological barriers that hindered the translation of fluorescence lifetime techniques to clinical settings are now being addressed and current results are very promising, one important challenge remains: the ability to effectively validate the fluorescence-derived parameters with standard histopathology-which is the current standard-of-care. There are two primary issues. The first is the coregistration problem. Once the optically investigated tissue is removed (biopsy or en bloc) from the patient, it undergoes shrinkage and deformations that are further enhanced by fixation and histologic processing. Thus, in many instances, the coregistration of optically interrogated areas with corresponding pathology is subject to spatial registration error. The second issue is the problem with standard histology itself. Tissue evaluation is based primarily on the assessment of tissue morphology rather than on metabolism or physiology. Thus, pathophysiological alterations potentially sensed by optical techniques cannot be directly validated. Therefore, other means to evaluate the tissue samples and validate optical results are needed.

\section{Label-Free Multimodal Multiphoton Imaging: Addressing the Need to Assess the Tumor Microenvironment, Not Just the Tumor Margin}

While most all of these intraoperative imaging technologies are used to evaluate the structural surgical margin created by the surgeon, or the tumor margin that represents the boundary between tumor cells and normal tissue, we recognize that molecular, metabolic, and functional physiological changes are concomitantly occurring within both the tumor and the tumor microenvironment. Increasing interest and research in understanding the dynamics of the tumor microenvironment have led to identifying it as one of the hallmarks of cancer. ${ }^{35,36} \mathrm{~A}$ recent Raman spectroscopy study of human breast cancer and the tumor micro- and macroenvironments revealed abnormal molecular signatures even centimeters away from the primary tumor site and different from the Raman signatures from human breast tissue from normal breast reduction surgeries. ${ }^{37}$ This study and studies involving exosome- and tumor-associated microvesicledriven intercellular communication ${ }^{38,39}$ suggest that tumorinduced molecular changes begin early in carcinogenesis, not only immediately beyond the structural tumor margin and into the tumor microenvironment but also more expansively throughout the entire organ and body. ${ }^{40}$ Building evidence suggests that these early systemic molecular changes precede tumor metastasis, and imaging and visualizing these early molecular and metabolic changes could potentially provide new early biomarkers of cancer, determine tumor aggressiveness, provide prognostic value, and possibly enable new targets for future therapeutic strategies.

To extend beyond label-free structural imaging of the surgical margin, multimodal multiphoton imaging techniques have been investigated for providing images based on molecular, metabolic, vibrational, or functional changes in tissue..$^{21,41,42}$ 
Recently, Tu et al. ${ }^{43}$ and Tu and Boppart ${ }^{44}$ developed a platform system based on a pumped photonic crystal fiber source that produced widely coherent supercontinuum suitable for pulse compression and shaping to efficiently drive label-free nonlinear processes of two- and three-photon autofluorescence, secondand third-harmonic generation, and broadband hyperspectral coherent anti-Stokes Raman scattering. ${ }^{45}$ This multimodal platform imaging system was able to simultaneously capture all channels for spatially and temporally correlated images of autofluorescent molecules associated with metabolism and cell function (NADH and FAD), microstructural organizational features of collagen and elastin, cellular and subcellular interfaces, and compositional molecular differences throughout tissue. Most interesting, Tu et al. ${ }^{46}$ realized a newly discovered ability to visualize exosomes and microvesicles being released from tumor cells and distributing throughout the micro/macroenvironment, along with concurrent changes in the metabolism of nontumor cells present in these environments. More recent (unpublished) results have demonstrated real-time in vivo imaging with this platform to reveal intravital dynamics of these tumor-associated exosomes and microvesicles, and a new portable imaging cart that has been used intraoperatively to image and characterize the microenvironment of freshly resected human breast masses, to quantify microvesicle density, and to correlate density and optical signatures with tumor grade and stage.

These results support the importance of the tumor micro/ macroenvironment and the roles that these play in carcinogenesis. The use of nonlinear imaging platforms and portable imaging systems, such as these, offers the potential for slide-free, stain-free, real-time, in vivo molecular histopathology. These results also lead to the provocative question that perhaps the structural tumor margin that has historically served as the gold standard by pathologists for determining tumor extent, greatly underestimates the molecular and metabolic extent and influence that the tumor exerts, far beyond what is structurally visible. In addition to a structural tumor margin, does a molecular tumor margin exist, or are these changes truly systemic, without a well-defined boundary? If there is a molecular tumor margin, can we define it, detect it, and locate it?

\section{Defining a Molecular Surgical Margin: a New Standard for Complete Surgical Resection of Tumor?}

These questions related to a molecular surgical margin are also being explored in a subset of head and neck tumors and oral premalignant lesions using a variety of label-free optical imaging techniques, including optical fluorescence imaging ${ }^{47}$ and narrowband imaging (NBI). ${ }^{48}$ In a study assessing the molecular divergence among tumors, conventional WL- and NBI-defined surgical margins for oral squamous cell carcinoma (OSCC) resection were defined through microarray analysis. ${ }^{48}$ Farah and colleagues showed that the surgical margins determined by NBI possess fewer molecular abnormalities than the more conservative surgical margins determined by WL examination. This finding provides a molecular foundation to the previously reported clinical evaluation of NBI and supports the hypothesis that resection to surgical margins that are determined by NBI rather than by WL examination leave less potentially malignant residual tissue and, thereby, increase the likelihood of a successful ablative surgery.
Although this study was limited to surface margins because of the nature of light penetration and absorption of NBI [using parallel beams of blue (400 to $430 \mathrm{~nm}$ ) and green (525 to $555 \mathrm{~nm}$ ) light], thus only highlighting mucosal surface texture and underlying vasculature, it does demonstrate the presence of, and the potential for, molecular margin identification and opens the door for development of other label-free optical approaches assessing deep margins. Further, it points to the possibility of incorporating multimodal approaches to detect molecular margins once developed; an area of research that is in its infancy but one that should be strategically targeted as it may hold the key to overcome some of the obstacles that have impeded the more widespread adoption of optical imaging techniques in oncological surgery.

Farah and colleagues have taken this approach one step further, undertaking different molecular assessments on OSCC surgical margins to test the robustness of the initial approach, which focused on mRNA signatures. In a follow-up study, ${ }^{49}$ they assessed the microRNA profile of tumor and WL- and NBI-defined surgical margins and identified a larger number of differentially expressed miRNA. Subsequently, they were able to create a spatially correlated integrated miRNA-mRNA signature interactome and then infer commonality in the miRNA-mRNA molecular interplay present in tumor tissue and in tissue identified as normal by conventional examination, at a level that is not apparent for tissue identified as normal by NBI. This again strongly supports the premise that resection of OSCC to surgical margins determined by NBI rather than WL leaves less potentially malignant residual tissue and increases the likelihood of surgical success.

Not only does this merging or blending of optical imaging technologies and molecular analyses assist in the definition and determination of molecular margins, it also allows a molecular understanding of the optical properties of tissues being imaged, thereby laying the foundation for studies assessing the clearance of surgical margins of resected tissues. ${ }^{50}$ Understanding the molecular basis of what a surgeon sees by implementing optical imaging techniques can facilitate adoption of such technologies and provide much needed evidence of the soundness of optical imaging approaches to often skeptical or hesitant clinicians. In a study aimed at elucidating the molecular pathways associated with fluorescence properties of oral potentially malignant disorders visualized under direct tissue autofluorescence, ${ }^{47}$ Farah and colleagues showed that each lesion type had a specific set of histology-related differentially expressed genes (DEGs), and that all tested samples shared a number of DEGs. Gene ontology enrichment revealed loss of fluorescence in epithelial hyperplasia was mostly due to changes in inflammation, cell cycle regulation, and apoptosis, while in epithelial dysplasia, loss of fluorescence was due to inflammation, angiogenesis, and extracellular matrix remodeling.

Given the known heterogeneity of tumors even from the same anatomical site, the aforementioned studies not only point to the challenge in determining molecular margins but simultaneously offer potential solutions that optical imaging approaches may bring to this domain. Building on the largescale genomic studies, which have characterized head and neck cancer, such as those undertaken by the Cancer Genome Atlas Network,${ }^{51}$ it is not only feasible to assess the global molecular profile of tumors but also to assess the genomic profile of single cell at either the tumor margin or the surgical bed with the introduction of single-cell sequencing. As molecular profiling of 
whole tumors, tissue specimens, and now individual cells is made possible, it will become increasingly important to couple optical imaging approaches with molecular signatures developed through such studies. This would be an effort to not only meet the clinical and regulatory requirements of comparison to the current gold standard of histopathology but also to leapfrog this tissue-based approach and supersede it with a single-cell molecularly based approach. Incorporation of data from single-cell sequencing studies into the design of multimodal multispectral optical imaging technologies may yet prove to be a viable solution that surgeons and pathologists seek, elevating optical imaging to a clinically useful tool that can simultaneously define the molecular margin, scan the surgical bed for individual residual tumor cells for removal, and provide confidence for a complete tumor resection.

\section{Other Label-Free Optical Imaging Technologies for Assessing the Surgical Margin}

In addition to these aforementioned technologies, several others exist that offer similar potential for slide-free, stain-free, realtime, in vivo imaging. Optical coherence tomography (OCT) has been demonstrated for imaging the in vivo tumor resection bed in human subjects during breast cancer surgery, yielding a sensitivity and specificity of $91.7 \%$ and $92.1 \%$, respectively, for detecting positive margins (Fig. 1), ${ }^{52}$ as well as for imaging the surgical margins of resected tumor specimens ${ }^{19,20,53-55}$ and loco-regional lymph nodes in human breast cancer surgeries. ${ }^{56}$ Related to OCT, optical coherence elastography provides 3-D maps of tissue mechanical properties, on the micrometer to millimeter scale. Here, a mechanical load is applied to tissue and the resultant displacement is measured using OCT. Images of strain or elastograms present identifying features based on mechanical contrast. ${ }^{57,58}$ Intraoperative Raman spectroscopy has been used to create molecular contrast maps of ex vivo specimen margins based on spectral features indicative of normal versus tumor tissues, ${ }^{59}$ and more recently, photoacoustic microscopy has recently been used to assess surgical specimens and margins, relying on inherent absorption contrast differences between normal and tumor tissues. ${ }^{60}$

\section{Perspectives from a Surgical Pathologist: Advancing Digital Pathology from Scanning Microscope Slides to Real-Time In Vivo Molecular Histopathology with New Contrasts and New Diagnostic Signatures}

There are significant impediments to the adoption of label-free optical imaging technologies in current oncological surgery. These barriers must be overcome for early adoption and translation into clinical practice. In addition to the technical limitations outlined above, these include barriers of time, cost, training, and an overall hesitation of surgeons and pathologists to adopt new technology as yet unproven in regards to clinical outcomes, despite the drive to diminish the need of toxic treatments, such as chemotherapy or radiotherapy, and improve mortality and morbidity of patients. These points highlight the importance of well-structured randomized controlled trials required to increase the level of evidence for the clinical utility of optical imaging technologies in intraoperative margin assessment.
Any new device must be easy to use, easy to learn, cost-effective, and deliver better clinical outcomes compared to current standard practice. New optical imaging devices must be scalable and capable of use in both resource-rich and resource-poor regions of the world. The ideal device must be able to provide the surgeon with a quick readout or easily interpretable result, with minimal reliance on additional expertise as surgeons appear to be hesitant in adoption of technologies that require interpretation of imaging data. Surgical theater space is always at a premium, so the addition of any large equipment and/or additional personnel within surgical theaters is a hindrance to adoption of new technology.

An ideal single modality device or combination platform should be able to scan large areas of tissue at high resolution within short periods of time, be ergonomic, accurate, and inexpensive to use. The device must be able to also interrogate deeper tissues to overcome the current limited penetration offered by most imaging modalities. Deep tissue interrogation is also relevant for the assessment of dysplasia at margins, which requires in situ cell-based imaging approaches and not just tissue-based surface scanning determination. Any advancement that combines broad-based imaging modalities with an ability to probe particular areas in depth would be advantageous and should be correlated with label-free molecular biomarkers that can be instantaneously interpreted by optical platforms.

As we continue to assess the mutational and genomic landscapes of tumors and construct molecular profiles and targeted signature panels for them, it is important that our thinking be geared toward assessment of a molecular margin and not just the cellular one. The expertise and role of the pathologist will become even more important and integrated with the use of these optical imaging technologies. Pathologists will play a central role by linking the newly generated genomic and molecular profiles associated with a molecular margin, with the cellular features that currently define the structural tumor margin. Likewise, as we begin to unravel the molecular profiles of individual cells and their dynamic contributions and interplay within tumors through single-cell sequencing, it may become possible to use these biomarker profiles as surrogates or targets for optical imaging strategies, which will guide clearance of the margin at the molecular level, and provide correlated histopathological outcomes.

\section{Conclusions and Focus on Future Directions}

As we come to understand the molecular heterogeneity of tumors and their margins, there was consensus at this conference that the gold standard is shifting, and developers should be working on approaches that leapfrog the current tissue-based gold standard and adopt a new paradigm of in vivo molecular histopathology, bypassing the perceived rigidity of current histopathological assessment methods and truly enacting the concept of optical biopsy.

Speakers and conference attendees recognized that new techniques and technologies should not be tethered to current standards but instead should be driving the creation and adoption of new ones. In the context of optical imaging for margin assessment, we should be espousing optical determinants of tissue clearance as the new gold standard for surgical success, as these at once will not only do away with the need for histopathological assessment of tissue resections but also offer the operating surgeon the ability to self-determine what constitutes 
a clear surgical margin. The challenge for developing new optical devices to enhance surgical margin delineation is that developers, surgeons, pathologists, and scientists must be continuously working together to integrate, implement, and adopt scientific advancements from disparate disciplines into a shared endeavor.

The concluding consensus on this topic was the importance of moving toward slide-free, stain-free, real-time, in vivo imaging of the surgical margin as well as the micro- and macrotissue environments surrounding the tumor. Points that resonated included the essential need to connect imaging modalities and imaging fields-of-view across multiple size scales, from mammography (x-ray and MRI) to microscopy (optical), which will require innovative solutions involving the acquisition, processing, and management of large volumes of 3-D image data and understanding how the underlying physics that generates image contrast correlate between different imaging modalities. With the enormous volumes of image data that can now be rapidly generated by not only optical but all biomedical imaging modalities in general, there will be an increasing need for automated CAD algorithms to rapidly screen images and flag suspicious ones. These CAD algorithms will likely be driven by more sophisticated machine- and deep-learning algorithms to identify underlying trends and patterns in the image data that cannot be visually identified by a human reader.

Finally, there will be a continual need for engineers and scientists to work collaboratively with surgeons and pathologists to not only identify the fundamental challenges encountered daily in surgery and medicine, but then also to critically advance the technological capabilities and prove their effectiveness through randomized controlled trials. These teams will need to work collaboratively toward adoption strategies that would allow these new imaging technologies to be introduced and integrated into both the individualized patient care as well as the greater logistical operations of the entire hospital and healthcare system. Collectively, there are mutual benefits to all parties, including more technologically capable imaging systems, more clinical utility of the systems and the added diagnostic and prognostic data and information that they generate, and ultimately the benefit to patients with improved surgical and health outcomes.

\section{Disclosures}

Stephen A. Boppart is a cofounder and chief medical officer of Diagnostic Photonics, Inc., which is developing intraoperative optical imaging systems based on interferometric synthetic aperture microscopy. $\mathrm{He}$ also receives royalties from the Massachusetts Institute of Technology from licensed patents related to OCT. J. Quincy Brown is a cofounder and chief research officer for Instapath, Inc. and has a financial interest in Zenalux Biomedical, Inc. Camile S. Farah is a recipient of educational and research grants from Olympus, Inc., for oncological research on NBI. Christobel M. Saunders is a minor shareholder in OncoRes, Inc., which is developing optical coherence elastography solutions for tissue diagnostics. All other authors have nothing to disclose.

\section{Acknowledgments}

We would like to thank all of our colleagues, physicians, researchers, students, staff, and patients who tirelessly engage in research to develop optical imaging technologies to improve human health. We also thank the organizers and sponsors of the 2017 International Conference on Biophotonics for their support of this effort and for offering to highlight these technologies to the international community. Finally, we thank SPIE for supporting the publication of this position paper in the Journal of Biomedical Optics. Funding to support the writing of this paper was provided in part by the National Institutes of Health (R01 CA213149 and R01 EB023232, S.A.B., and R01 CA187427 and R21 CA178578, L.M.). Research presented in this paper was partly funded by the Queensland Government Smart Futures Co-Investment Fund, Royal Brisbane and Women's Hospital Foundation, and Cancer Australia awarded to C.S.F.

\section{References}

1. International Conference on Biophotonics, April 30-May 1, 2017, Fremantle, Western Australia, http://icob2017.com/.

2. H. Silberman and A. W. Silberman, Principles and Practice of Surgical Oncology: A Multidisciplinary Approach to Difficult Problems, Wolters Kluwer Health, Philadelphia (2012).

3. J. M. Jorns et al., "Intraoperative frozen section analysis of margins in breast conserving surgery significantly decreases reoperative rates: oneyear experience at an ambulatory surgical center," Am. J. Clin. Pathol. 138, 657-669 (2012).

4. H. A. Amer et al., "Digital breast tomosynthesis versus full-field digital mammography — which modality provides more accurate prediction of margin status in specimen radiography?" Eur. J. Radiol. 93, 258-264 (2017).

5. F. D'Halluin et al., "Intra-operative touch preparation cytology following lumpectomy for breast cancer: a series of 400 procedures," Breast 18, 248-253 (2009).

6. F. Schnabel et al., "Randomized prospective study of lumpectomy margin assessment with use of MarginProbe in patients with nonpalpable breast malignancies," Ann. Surg. Oncol. 21, 1589-1595 (2014).

7. Q. R. Tummers et al., "Real-time intraoperative detection of breast cancer using near-infrared fluorescence imaging and methylene blue," Eur. J. Surg. Oncol. 40, 850-858 (2014).

8. Y. Urano et al., "Rapid cancer detection by topically spraying a $\gamma$-glutamyltranspeptidase-activated fluorescent probe," Sci. Transl. Med. 3, 110 ra119 (2011).

9. F. L. Tansi et al., "Potential of activatable FAP-targeting immunoliposomes in intraoperative imaging of spontaneous metastases," Biomaterials 88, 70-82 (2016).

10. Y. Liu et al., "Hands-free, wireless goggles for near-infrared fluorescence and real-time image-guided surgery," Surgery 149, 689-698 (2011).

11. C. Higgins, "Applications and challenges of digital pathology and whole slide imaging," Biotech. Histochem. 90, 341-347 (2015).

12. FDA News Release, "FDA allows marketing of first whole slide imaging system for digital pathology," https://www.fda.gov/NewsEvents/ Newsroom/PressAnnouncements/ucm552742.htm (12 April 2017).

13. H. Ballal et al., "Predictors of re-excision in wire-guided wide local excision for early breast cancer: a Western Australian multi-centre experience," ANZ J. Surg. 85, 540-545 (2015).

14. M. R. Decker et al., "The role of intraoperative pathologic assessment in the surgical management of ductal carcinoma in situ," Ann. Surg. Oncol. 23, 2788-2794 (2016).

15. S. Abeytunge et al., "Confocal microscopy with strip mosaicing for rapid imaging over large areas of excised tissue," J. Biomed. Opt. 18, 061227 (2013).

16. S. Abeytunge et al., "Rapid confocal imaging of large areas of excised tissue with strip mosaicing," J. Biomed. Opt. 16, 050504 (2011).

17. D. S. Gareau et al., "Confocal mosaicing microscopy in Mohs skin excisions: feasibility of rapid surgical pathology," J. Biomed. Opt. 13, 054001 (2008).

18. S. Abeytunge et al., "Evaluation of breast tissue with confocal stripmosaicking microscopy: a test approach emulating pathology-like examination," J. Biomed. Opt. 22, 034002 (2017).

19. N. Iftimia et al., "Handheld optical coherence tomography-reflectance confocal microscopy probe for detection of basal cell carcinoma and delineation of margins," J. Biomed. Opt. 22, 076006 (2017). 
20. M. G. Giacomelli et al., "Virtual hematoxylin and eosin transillumination microscopy using epi-fluorescence imaging," PLoS One 11, e0159337 (2016).

21. T. Yoshitake et al., "Direct comparison between confocal and multiphoton microscopy for rapid histopathological evaluation of unfixed human breast tissue," J. Biomed. Opt. 21, 126021 (2016).

22. A. K. Glaser et al., "Light-sheet microscopy for slide-free non-destructive pathology of large clinical specimens," Nat. Biomed. Eng. 1, 0084 (2017).

23. M. Wang et al., "Gigapixel surface imaging of radical prostatectomy specimens for comprehensive detection of cancer-positive surgical margins using structured illumination microscopy," Sci. Rep. 6, 27419 (2016).

24. K. N. Elfer et al., "DRAQ5 and eosin ('D\&E') as an analog to hematoxylin and eosin for rapid fluorescence histology of fresh tissues," PLoS One 11, e0165530 (2016).

25. R. Reddy and M. Haris, "Imaging technologies from bench to bedside," J. Transl. Med. 13, 97 (2015).

26. A. C. Croce and G. Bottiroli, "Autofluorescence spectroscopy and imaging: a tool for biomedical research and diagnosis," Eur. J. Histochem. 58, 2461 (2014).

27. L. Marcu, P. M. W. French, and D. S. Elson, Fluorescence Lifetime Spectroscopy and Imaging: Principles and Applications in Biomedical Diagnostics, CRC Press Taylor \& Francis, London, United Kingdom (2014).

28. D. Gorpas et al., "Real-time visualization of tissue surface biochemical features derived from fluorescence lifetime measurements," IEEE Trans. Med. Imaging 35, 1802-1811 (2016).

29. D. Ma et al., "Technique for real-time tissue characterization based on scanning multispectral fluorescence lifetime spectroscopy (ms-TRFS)," Biomed. Opt. Express 6, 987-1002 (2015).

30. L. L. de Boer et al., "Fat/water ratios measured with diffuse reflectance spectroscopy to detect breast tumor boundaries," Breast Cancer Res. Treat. 152, 509-518 (2015).

31. J. W. Spliethoff et al., "Real-time in vivo tissue characterization with diffuse reflectance spectroscopy during transthoracic lung biopsy: a clinical feasibility study," Clin. Cancer Res. 22, 357-365 (2016).

32. D. J. Evers et al., "Diffuse reflectance spectroscopy: towards clinical application in breast cancer," Breast Cancer Res. Treat. 137, 155-165 (2013).

33. R. Nachabé et al., "Estimation of biological chromophores using diffuse optical spectroscopy: benefit of extending the UV-VIS wavelength range to include 1000 to 1600 nm," Biomed. Opt. Express 1, 1432-1442 (2010).

34. H. J. C. M. Sterenborg et al., "Hyperspectral imaging for intraoperative margin assessment during breast cancer surgery," in Asia Communications and Photonics Conf. 2016, OSA Technical Digest, Optical Society of America (2016).

35. D. Hanahan and R. A. Weinberg, "The hallmarks of cancer," Cell 100, 57-70 (2000).

36. D. Hanahan and R. A. Weinberg, "Hallmarks of cancer: the next generation," Cell 144, 646-674 (2011).

37. S. You et al., "Raman spectroscopic analysis reveals abnormal fatty acid composition in tumor micro- and macroenvironments in human breast and rat mammary cancer," Sci. Rep. 6, 32922 (2016).

38. S. Kamerkar et al., "Exosomes facilitate therapeutic targeting of oncogenic KRAS in pancreatic cancer," Nature 546, 498-503 (2017).

39. S. K. Gopal et al., "Extracellular vesicles: their role in cancer biology and epithelial-mesenchymal transition," Biochem. J. 474, 21-45 (2017).

40. M. D. Cruz et al., "Metabolic reprogramming of the premalignant colonic mucosa is an early event in carcinogenesis," Oncotarget 8, 20543-20557 (2017).

41. T. Meyer et al., "Multimodal nonlinear microscopic investigations on head and neck squamous cell carcinoma: toward intraoperative imaging," Head Neck 35, E280-E287 (2013).

42. M. Ji et al., "Detection of human brain tumor infiltration with quantitative stimulated Raman scattering microscopy," Sci. Transl. Med. 7, 309ra163 (2015).

43. H. Tu et al., "Scalar generalized nonlinear Schrodinger equationquantified continuum generation in an all-normal dispersion photonic crystal fiber for broadband coherent optical sources," Opt. Express 18, 27872-27884 (2010).
44. H. Tu and S. A. Boppart, "Coherent fiber supercontinuum for biophotonics," Laser Photonics Rev. 7, 628-645 (2013).

45. H. Tu et al., "Stain-free histopathology by programmable supercontinuum pulses," Nat. Photonics 10, 534-540 (2016).

46. H. Tu et al., "Concurrence of extracellular vesicle enrichment and metabolic switch visualized label-free in the tumor microenvironment," Sci. Adv. 3, e1600675 (2017).

47. F. Kordbacheh, N. Bhatia, and C. S. Farah, "Patterns of differentially expressed genes in oral mucosal lesions visualized under autofluorescence (VELscope ${ }^{\mathrm{TM}}$ )," Oral Dis. 22, 285-296 (2016).

48. C. S. Farah et al., "Improved surgical margin definition by narrow band imaging for resection of oral squamous cell carcinoma: a prospective gene expression profiling study," Head Neck 38, 832-839 (2016).

49. C. S. Farah, S. A. Fox, and A. J. Dalley, "Integrated miRNA-mRNA spatial signature for oral squamous cell carcinoma: a prospective profiling study of narrow band imaging guided resection," Sci. Rep. (in press) (2017).

50. C. S. Farah et al., "Molecular classification of autofluorescence excision margins in oral potentially malignant disorders," Oral Dis. (in press) (2017).

51. The Cancer Genome Atlas Network, "Comprehensive genomic characterization of head and neck squamous cell carcinomas," Nature 517, 576-582 (2015).

52. S. J. Erickson-Bhatt et al., "Real-time imaging of the resection bed using a handheld probe to reduce incidence of microscopic positive margins in cancer surgery," Cancer Res. 75, 3706-3712 (2015).

53. F. T. Nguyen et al., "Intraoperative evaluation of breast tumor margins with optical coherence tomography," Cancer Res. 69(22), 8790-8796 (2009).

54. A. M. Zysk et al., "Intraoperative assessment of final margins with a handheld optical imaging probe during breast conserving surgery may reduce the reoperation rate: results of a multi-center study," Ann. Surg. Oncol. 22, 3356-3362 (2015).

55. P. L. Hsiung et al., "Benign and malignant lesions in the human breast depicted with ultrahigh resolution and three-dimensional optical coherence tomography," Radiology 244, 865-874 (2007).

56. R. M. Nolan et al., "Intraoperative optical coherence tomography for assessing human lymph nodes for metastatic cancer," BMC Cancer 16, 144 (2016).

57. K. M. Kennedy et al., "Quantitative micro-elastography: imaging of tissue elasticity using compression optical coherence elastography," Sci. Rep. 5, 15538 (2015).

58. W. M. Allen et al., "Wide-field optical coherence micro-elastography for intraoperative assessment of human breast cancer margins," Biomed. Opt. Express 7, 4139-4153 (2016).

59. S. Kennedy et al., "Correlation of breast tissue histology and optical signatures to improve margin assessment techniques," J. Biomed. Opt. 21, 066014 (2016).

60. T. T. W. Wong et al., "Fast label-free multilayered histology-like imaging of human breast cancer by photoacoustic microscopy," Sci. Adv. 3, e1602168 (2017).

Stephen A. Boppart is an Abel Bliss professor of engineering in the Departments of Electrical and Computer Engineering, Bioengineering, and Medicine, a head of the Biophotonics Imaging Laboratory, and a director of the Center for Optical Molecular Imaging at the Beckman Institute for Advanced Science and Technology. His interests include the development of optical imaging technologies for medical applications, with emphasis on translating these to clinical applications. He is a fellow of SPIE, IEEE, AAAS, OSA, BMES, and AIMBE.

J. Quincy Brown is the Paul H. and Donna D. Flower assistant professor in the Department of Biomedical Engineering at Tulane University, and a program member in the Tulane Cancer Center. His research interests include point-of-care digital pathology for cancer diagnosis. He is a member of SPIE, OSA, IEEE-EMBS, and BMES.

Camile S. Farah is clinician-scientist and expert in oral oncological translational research. His clinical and laboratory work with optical fluorescence imaging and narrow band imaging has set the new gold standard for head and neck cancer endoscopy, resection margin delineation, and detection of oral potentially malignant lesions. His lab has explored diagnostic biomarker signatures for oral premalignant 
lesions, predictive biomarker profiles for oral cancer progression, and a multigene discriminatory biomarker panel for oral squamous cell carcinoma.

Esther Kho received her MSc degree in biomedical engineering from the University of Groningen, The Netherlands, in 2014. Since 2015, she has been working towards the PhD in the Department of Surgery at the Netherlands Cancer institute/Antoni van Leeuwenhoek Hospital, Amsterdam, The Netherlands. Her research involves the development, testing and clinical evaluation of a hyperspectral imaging system specifically for the assessment of tumor positive resection margins during breast conserving surgery.

Laura Marcu is a professor of biomedical engineering and neurological surgery, and a head of the Biophotonics Laboratory at the University of California Davis. Her research interests include the Development of Optical Spectroscopy and Imaging Technologies, with emphasis on image-guided interventions, intravascular diagnostics, and regenerative medicine. She is a fellow of SPIE, OSA, AIMBE, and BMES.
Christobel M. Saunders is internationally recognized as one of Australia's most prominent research-orientated cancer surgeons. She has substantially contributed to many clinical aspects of breast cancer research including clinical trials of new treatments, psychosocial, translational and health services research. In the past five years, she has published 100 peer-reviewed journal articles and is currently WA Scientist of the Year. She sits on boards of a number of cancer organizations including the ANZ Breast Cancer Trials Group, and is a president of the Breast Surgical Society of Australia and New Zealand.

Henricus J. C. M. Sterenborg has a background in applied physics and has been active in biomedical optics research since 1987 as a staff member of the Medical Laser Centre of the Academic medical Centre in Amsterdam. In 1998, he (co)founded the Centre for Optical Diagnostics and Therapy at the Erasmus Medical Center and in 2008 he was appointed professor of photodynamic therapy. Since 2013 he has held a joint position at the Department of Biomedical Engineering and Physics at the Academic Medical Centre in Amsterdam and the Surgical Innovations Group at the Netherlands Cancer Institute. 